\title{
Correction to: The potential use of tideglusib as an adjuvant radio-therapeutic treatment for glioblastoma multiforme cancer stem-like cells
}

\author{
Jolie Bou-Gharios ${ }^{1,2}$. Sahar Assi ${ }^{1} \cdot$ Hisham F. Bahmad ${ }^{1,3}$. Hussein Kharroubi ${ }^{1} \cdot$ Tarek Araji $^{1} \cdot$ Reda M. Chalhoub ${ }^{1,4}$. \\ Farah Ballout ${ }^{1} \cdot$ Hayat Harati ${ }^{2}$. Youssef Fares ${ }^{2} \cdot$ Wassim Abou-Kheir $^{1}$ (D)
}

Published online: 1 December 2020

(c) Maj Institute of Pharmacology Polish Academy of Sciences 2020

Correction to: Pharmacological Reports (2020) https://doi.org/10.1007/s43440-020-00180-5

The original version of this article, published on November 2, 2020, contained a mistake.

The spelling of Youssef Fares' name was incorrect in this article. The original article has been corrected.

The original article can be found online at https://doi.org/10.1007/ s43440-020-00180-5.

Youssef Fares

yfares@ul.edu.lb

$\triangle$ Wassim Abou-Kheir

wa12@aub.edu.lb

1 Department of Anatomy, Cell Biology and Physiological Sciences, Faculty of Medicine, American University of Beirut, Bliss Street, DTS Bldg, Room 116-B, Riad el Solh, PO Box 110236/41, Beirut 1107-2020, Lebanon

2 Neuroscience Research Center, Lebanese University Faculty of Medicine, Beirut, Lebanon

3 Present Address: Arkadi M. Rywlin M.D. Department of Pathology and Laboratory Medicine, Mount Sinai Medical Center, Miami Beach, FL, USA

4 Present Address: Medical Scientist Training Program, College of Medicine, Medical University of South Carolina, Charleston, SC, USA 